\title{
Sky cover from MFRSR observations
}

\author{
E. Kassianov, J. C. Barnard, L. K. Berg, C. Flynn, and C. N. Long \\ Pacific Northwest National Laboratory, Richland, Washington, 99352, USA \\ Received: 28 December 2010 - Published in Atmos. Meas. Tech. Discuss.: 24 January 2011 \\ Revised: 29 June 2011 - Accepted: 8 July 2011 - Published: 20 July 2011
}

\begin{abstract}
The diffuse all-sky surface irradiances measured at two nearby wavelengths in the visible spectral range and their modeled clear-sky counterparts are the main components of a new method for estimating the fractional sky cover of different cloud types, including cumuli. The performance of this method is illustrated using 1-min resolution data from a ground-based Multi-Filter Rotating Shadowband Radiometer (MFRSR). The MFRSR data are collected at the US Department of Energy Atmospheric Radiation Measurement (ARM) Climate Research Facility (ACRF) Southern Great Plains (SGP) site during the summer of 2007 and represent 13 days with cumuli. Good agreement is obtained between estimated values of the fractional sky cover and those provided by a well-established independent method based on broadband observations.
\end{abstract}

\section{Introduction}

Cloud fraction (CF) is a key cloud property that controls the amount of transmitted and reflected solar radiation, and thus the radiation balance at the surface (e.g., Dong et al., 2006; Berg et al., 2011). Aerosols may substantially change the CF, and these changes are thought to be sensitive to the aerosol optical depth (AOD) and the single-scattering albedo (SSA) (Perlwitz and Miller, 2010). The strength and even sign of the CF-AOD relationship have been controversial for at least a decade (e.g., Quaas et al., 2010, and references therein) and such controversy has resulted partially from sampling issues. For example, the aerosol and cloud properties of interest are obtained typically with different spatial/temporal resolution by several different surface and spaceborne instruments. Thus, there is an essential need to have a tool that can offer the coincident and collocated measurements and retrievals of cloud and aerosol properties.

Correspondence to: E. Kassianov

(evgueni.kassianov@pnl.gov)

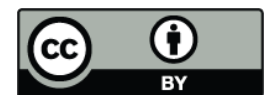

Kaufman and Koren (2006) applied data from the Aerosol Robotic Network (AERONET) for quantifying the effect of pollution and smoke aerosols on the directional CF, defined as a fraction of time that a ground-based sunphotometer would detect a cloud for a given direction from the instrument to the sun. The data were collected around the globe and subdivided into continental, coastal/oceanic, and biomass burning subsets. They found an increase in the directional CF of water clouds with an increase in AOD and decrease of the aerosol absorption, and also demonstrated that this relationship is not a function of aerosol type and location. Similarly to Kaufman and Koren (2006), Lane et al. (2002) estimated the directional CF of small, scattered cumuli from Multi-Filter Rotating Shadowband Radiometer (MFRSR) observations at the US Department of Energy Atmospheric Radiation Measurement (ARM) Climate Research Facility Southern Great Plains (SGP) site and showed that typical CF values do not exceed 0.3. However, the impact of aerosol on the CF was not considered.

The majority of the climate-related studies (e.g., Zhang et al., 2010) have used the fractional sky cover $N$, which is a hemispherical measure of cloud amount and is defined as the fraction of hemispherical sky covered by clouds (e.g., Hahn et al., 2001). The estimation of $N$ can be performed using empirical method (e.g., Long et al., 2006). This method uses measured shortwave, broadband all-sky fluxes and their inferred clear-sky counterparts, and provides "shortwave" sky cover $N_{\mathrm{SW}}$. Here we illustrate how spectrally resolved fluxes in the visible spectral range can be applied for estimating $N$. Since cumuli appear to be more susceptible to the aerosol changes in comparison with other low clouds ( $\mathrm{Su}$ et al., 2010), this estimation is illustrated for days with cumuli and typical aerosol loading. For these selected days, the aerosol optical properties (e.g., AOD and SSA) are consistent with the climatological values (e.g., McComiskey et al., 2008; Michalsky et al., 2010).

Published by Copernicus Publications on behalf of the European Geosciences Union. 


\section{Approach}

Originally, Min et al. (2008) suggested a technique for estimating $N$ from the MFRSR data. This technique involves the ratio of MFRSR-measured diffuse transmittance at two wavelengths ( 870 and $415 \mathrm{~nm}$ ), the so-called the transmittance ratio. The diffuse transmittance was defined as a diffuse flux divided by the corresponding extraterrestrial spectral irradiance inferred from Langley regression on clear-sky days. The technique requires the clear- and cloudy-sky baselines. To obtain them, sufficiently long periods with optically thick clouds are required. Such periods can be obtained easily for clouds with large horizontal extent such as stratus/stratocumulus clouds. On the other hand, cumuli may be optically thin and have more limited spatial extent. The corresponding cloudy-sky transmittance ratio has smallscale fluctuations (Fig. 1c), which hamper estimation of the cloudy-sky baseline. Thus, application of this technique to cumuli could be problematic. Moreover, the application of this technique is complicated by issues of diurnal changes of aerosol properties. As an example, we consider a day with strong diurnal AOD variations where "evening" AOD values (from 17:00 to 18:00 CST) exceed their "morning" counterparts (from 09:00 to 10:00 CST) by more than two times (Fig. 1a). As a result, the obtained "morning" clear-sky baseline is not representative of the "evening" period (Fig. 1c) and vice versa.

For a given solar zenith angle, the temporal variations of the clear-sky fluxes are mostly governed by changes of aerosol properties, and these changes should be considered. We apply a three-step physically-based approach (Kassianov et al., 2011) using: (i) the spectrally resolved direct and diffuse irradiances for retrieval of aerosol optical properties during clear sky periods (Harrison and Michalsky, 1994; Kassianov et al., 2007), (ii) temporal interpolation of the retrieved clear-sky aerosol properties for temporally "nearby" cloudy intervals, and (iii) calculations the clear-sky fluxes by using a radiative transfer model (Kassianov and Kogan, 2002) and the aerosol properties found by the temporal interpolation. By "nearby" we mean cloudy periods, during which we cannot find aerosol optical properties. These periods are sandwiched between clear periods, when it is possible to obtain aerosol optical properties. These properties include the AOD, and vertically-integrated SSA and asymmetry parameter. Figure 1c shows an application of this approach for obtaining a "clear-sky" baseline for the difference of the measured diffuse all-sky fluxes at two wavelengths (415 and $500 \mathrm{~nm}$ ). We apply this difference to define $N$ as follows.

In the framework of plane-parallel approximation, all-sky diffuse fluxes can be described as

$F(\lambda)=(1-N) F_{0}(\lambda)+N F_{1}(\lambda)$,

where $F_{0}$ and $F_{1}$ is the clear-sky and cloudy-sky diffuse flux, respectively. Obviously, this approximation is not able to
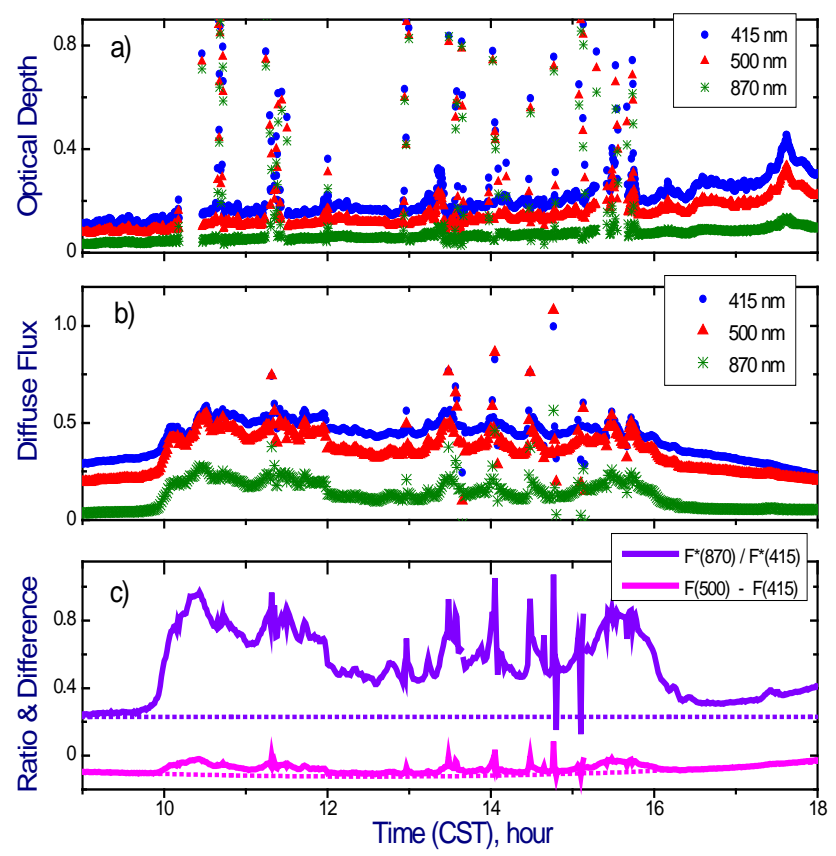

Fig. 1. Temporal realizations of optical depth at three wavelengths $(415,500$, and $870 \mathrm{~nm})(\mathbf{a})$, the corresponding all-sky diffuse fluxes (b), and the diffuse transmittance ratio and difference of diffuse fluxes (c) for 28 July 2007 . Violet dotted and magenta dotted lines (c) represent the "clear-sky" baselines for the diffuse transmittance ratio and diffuse flux difference, respectively.

explain the large fluctuations of the observed diffuse fluxes (Fig. 1b). Note that these fluctuations are strongly correlated at 415 and $500 \mathrm{~nm}$ wavelengths (Fig. 1b). Consequently, the cloud-induced contribution to the all-sky diffuse irradiance is proportional at these wavelengths. As a result, the difference of irradiances (Fig. 1c) has fluctuations with much smaller amplitude relative to the corresponding all-sky spectral fluxes (Fig. 1b). This suggests that in comparison with the spectral diffuse fluxes (415 and $500 \mathrm{~nm}$ ), this difference is less sensitive to the cloud-induced effects. Thus, for estimating $N$ we apply the difference that can be written as

$$
\begin{aligned}
F(500) & -F(415)=(1-N)\left[F_{0}(500)-F_{0}(415)\right] \\
& +N\left[F_{1}(500)-F_{1}(415)\right]
\end{aligned}
$$

The right part of Eq. (2a) has two terms, which represent $\mathrm{N}$ weighted contributions of the clear-sky and cloudy-sky components to the difference of diffuse fluxes. For a given sky cover $(N)$, the sign and magnitude of these contributions are defined by spectral changes of the extraterrestrial spectral irradiance, solar zenith angle, cloud and aerosol properties and surface albedo.

Typically, the spectral changes of AOD are substantial in the visible spectral range and these changes are mostly responsible for considerable spectral variations of the clear-sky diffuse fluxes at a given time. In contrast to AOD, cloud 

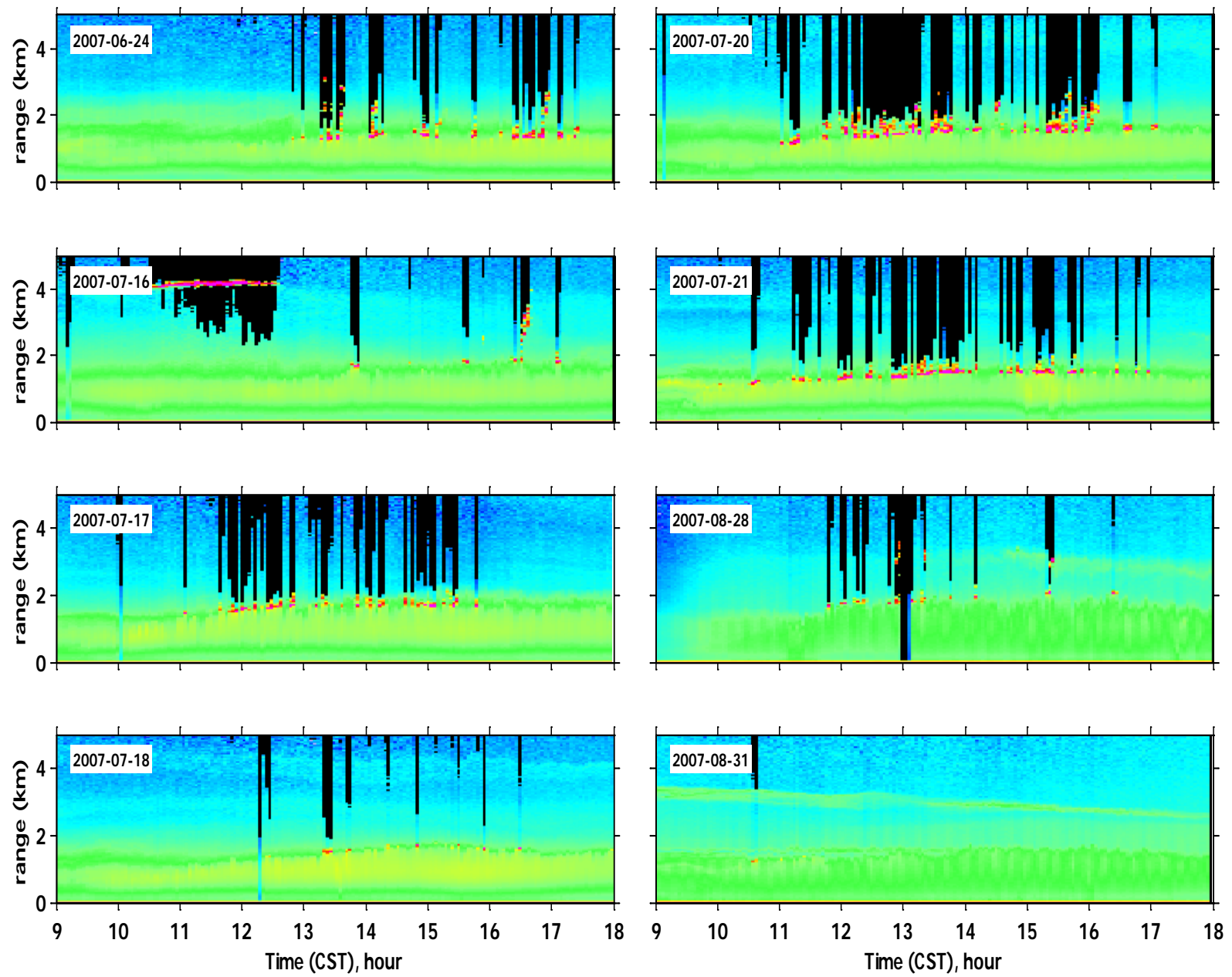

Fig. 2. Two-dimensional images of ground-based micropulse attenuated lidar backscatter for the 8 days when TSI observations are available.

optical properties do not vary much with wavelength in the visible region, and compared to the clear-sky diffuse fluxes at 415 and $500 \mathrm{~nm}$, their cloudy-sky counterparts are comparable. Therefore, to a first approximation, we can neglect the second right term of the Eq. (2a) and obtain

$F(500)-F(415) \approx(1-N)\left[F_{0}(500)-F_{0}(415)\right]$

From Eqs. (2b) we define the "visible" fractional sky cover as

$N_{\text {vis }} \approx 1-[F(500)-F(415)] /\left[F_{0}(500)-F_{0}(415)\right]$

Equation (3) includes a normalized difference of the all-sky diffuse fluxes. Such normalization removes the solar zenith angle effects and potential observational biases. Since this difference appears less sensitive to the cloud-induced effects relative to the corresponding spectral diffuse fluxes, Eq. (3) could be applicable for different cloud types, including stratiform clouds with large horizontal extent and cumuli with strong temporal/spatial variations of geometrical and optical properties.
We note that Eq. (3) does not require additional information on cloud type. Thus, the proposed retrieval has potential to be used operationally, with minimal human intervention for conditions with a wide range of cloud types. Certainly, its application is limited to cases where the cloudysky contribution to the difference of all-sky diffuse fluxes (see Eq. (2a)) is small relative to its clear-sky counterpart. Obvious exceptions include conditions when: (i) the AOD is almost spectrally independent (e.g., Slingo et al., 2006), (ii) cloud optical depth (COD) is small and comparable with AOD (e.g., Dupont et al., 2008), and (iii) different combinations of the preceding two factors. Conditions (i) and (ii) represent an aerosol layer with significant fraction of large particles (e.g., dust), and optically thin cirrus clouds (e.g., semitransparent), respectively. Another potential issue could arise with lasting overcast conditions when aerosol retrievals are very limited for a day of interest and therefore, estimation of the clear sky irradiance is problematic. For such conditions, aerosol properties obtained for the "nearby" day could be applied for such estimation. A similar approach is used 


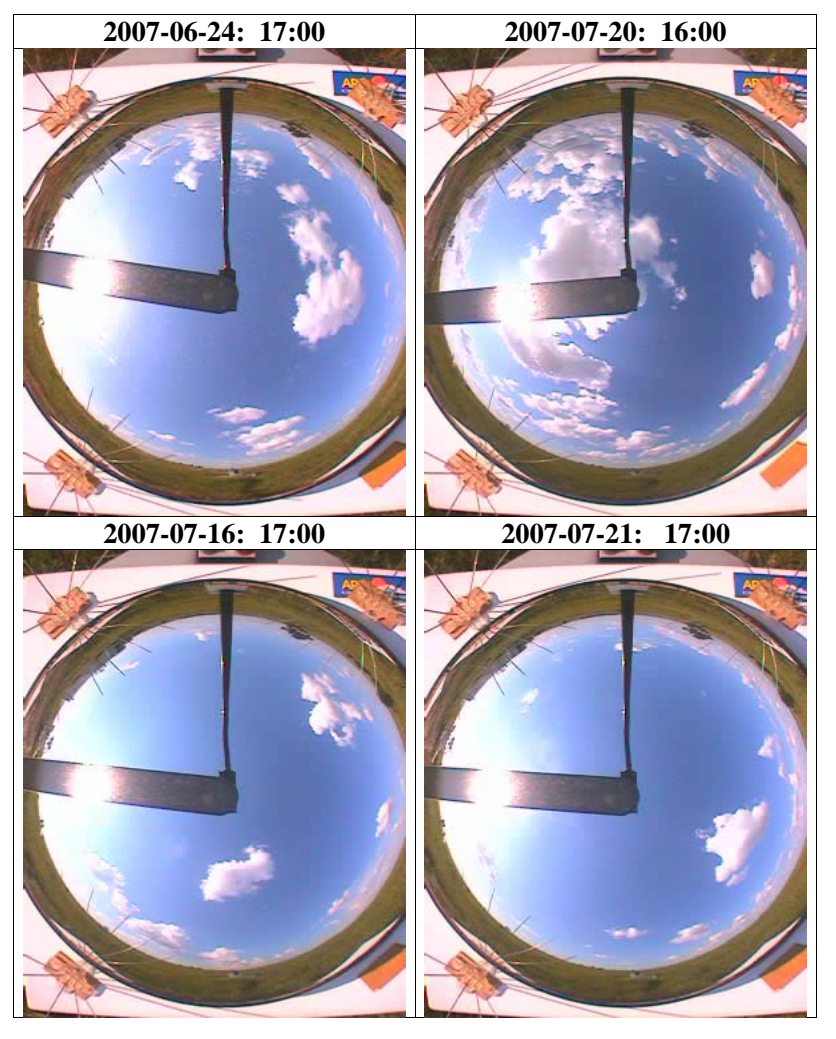

Fig. 3a. Hemispherical total sky images for the first 4 days in Fig. 2. The local time is included.

by the well-established retrieval of Long et al. (2006). Section 4 illustrates an application of the retrieval and contains the comparison of $N_{\text {vis }}$ with independent data. Before we embark on this application and comparison, instruments and data for comparison will be described.

\section{Observations}

We select 13 days with cumuli observed during summer 2007 at the ARM SGP site following the method described by Berg and Kassianov (2008). To illustrate the flexibility of our approach, we include one day (16 July 2007) with a transition from an almost completely overcast mid-latitude cloud layer in the morning to low-latitude cumuli in the afternoon. The site is equipped with numerous instruments for sampling cloud, aerosol and radiative properties. In particular, the ARM Active Remotely Sensed Clouds Locations (ARSCL) value-added product combines measurements of the cloud radar, laser ceilometers, microwave radiometer and micropulse lidar, and provides the best estimates of cloud boundaries. These instruments have a narrow field of view (FOV) and detect clouds observed directly above them (for example, Fig. 2 shows lidar observations). Thus, the ASRCL-based cloud properties (e.g., cloud frac-

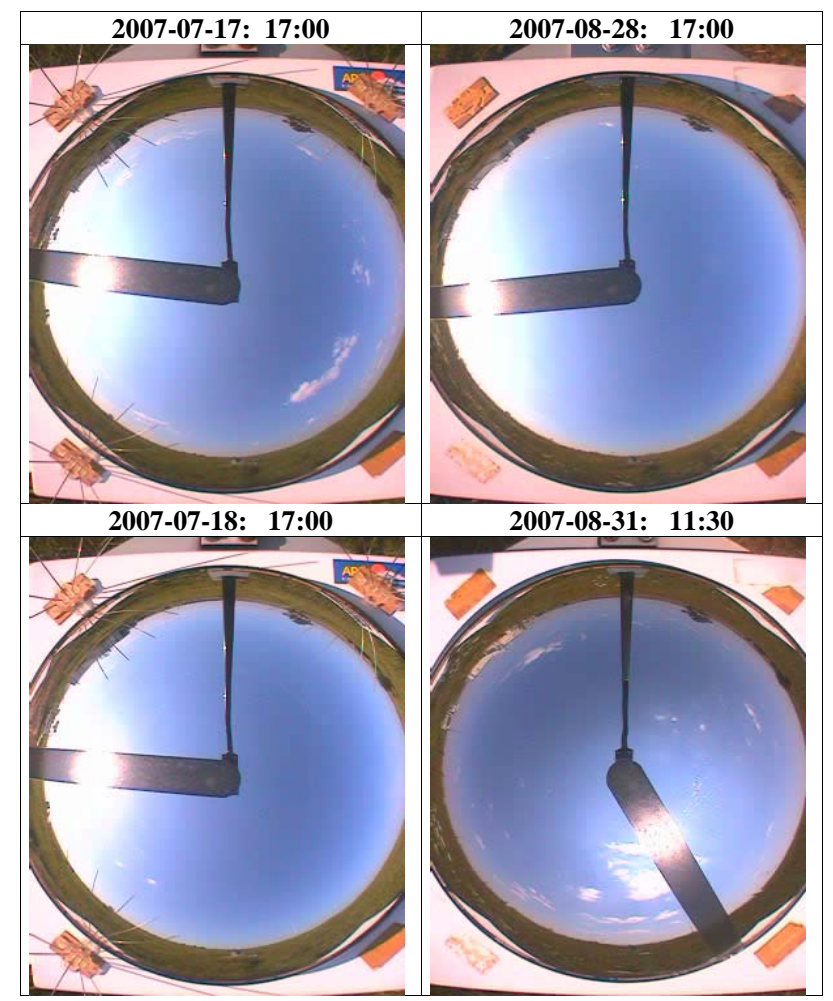

Fig. 3b . Hemispherical total sky images for the last 4 days in Fig. 2.

tion) may not be representative of those obtained for a region surrounding these instruments. In our analysis, we use the ASRCL-based cloud fraction for low clouds (cloud base is less than $3 \mathrm{~km}$ ) and for all clouds. In addition to the ARSCL measurements, we apply collocated and coincident observations of the total sky imager (TSI). The TSI has a hemispherical FOV and routinely provides time series of hemispherical sky images for a large area neighboring the ACRF site (Fig. 3). The difference between $N_{\mathrm{SW}}$ and $N_{\text {vis }}$ can be attributed by several factors, including the vertical stratification of clouds and their horizontal distribution over a given area. Here the ARSCL-based cloud fraction (as function of cloud base) and the TSI images are applied primarily to examine the sensitivity of this difference to these factors.

The MFRSRs measure the total all-sky surface downwelling irradiance and its diffuse and direct components at wavelengths of 415, 500, 615, 673, 870, and $940 \mathrm{~nm}$ (visible and near-IR spectral regions) with 20-sec temporal resolution (Harrison and Michalsky, 1994). We averaged the 20-sec MFRSR data over 1-min interval and used 1-min averages in our analysis. The high-temporal resolution MFRSR observations at the ACRF site allow one to capture the small-scale cloud-induced fluctuations of optical depth (Fig. 1a): instances with "spikes" in the optical depth define events when a cloud blocks the direct solar beam and the total optical depth is a sum of AOD and COD. The observed large sharp 


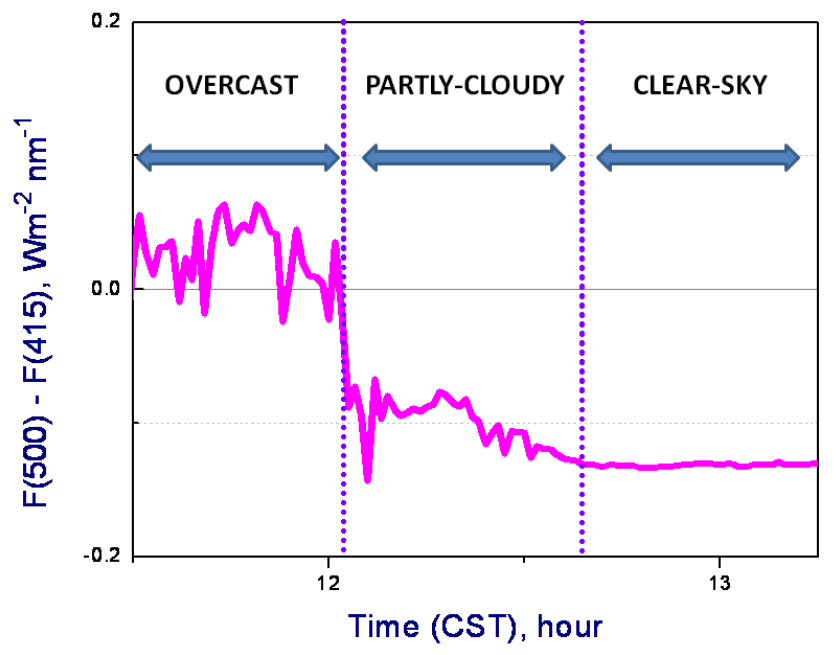

Fig. 4. Difference of diffuse fluxes at two wavelengths (415 and $500 \mathrm{~nm}$ ) as function of time for 16 July 2007 . The completely overcast cloudy (from 11:30 to 12:05 CST) and clear-sky (from 12:65 to 13:25 CST) are shown. The corresponding values of the fractional sky cover are 0.0 and 1.0. The difference is positive and negative for the overcast and clear-sky periods, respectively. On the average, the absolute values of the clear-sky difference are almost three times larger than their overcast counterparts.

changes of the diffuse irradiance (Fig. 1b) from smooth to rough are due to clouds, and changes of their geometrical and optical properties.

Analysis of time series of the TSI images and the MFRSRmeasured diffuse irradiances reveals that the observed difference $F(500)-F(415)$ has positive and negative values for the completely overcast cloudy $(N=1)$ and clear-sky $(N=0)$ conditions, respectively (Fig. 4). Thus, the corresponding sign change in this difference can be considered as a simple indicator of switching from a partly-cloudy sky to overcast sky. Also, this analysis reveals that the largest positive values occur for optically thin clouds (cloud images are bright) while the smallest positive values observed for optically thick clouds (cloud images are dark). Figure 4 shows the difference obtained for a day when the sky was almost completely overcast with optically thin clouds around noon. The corresponding average value of the overcast difference $F_{1}(500)-F_{1}(415)$ is about 0.03 . The well-known weak spectral dependence of cloud optical properties in the visible spectral range is mainly responsible for the small values of the overcast difference. In contrast, a strong spectral dependence of the aerosol optical properties (e.g., AOD) in the visible spectral range is mainly responsible for the relatively large values of the clear-sky difference $F_{0}(500)-F_{0}(415)$. From Fig. 4 one can conclude that the overcast value (0.03) is about four times smaller than the absolute value of its clearsky counterpart (0.13). Note that the latter demonstrates small day-to-day variations. For time periods with optically thick clouds, the overcast difference is even smaller (about

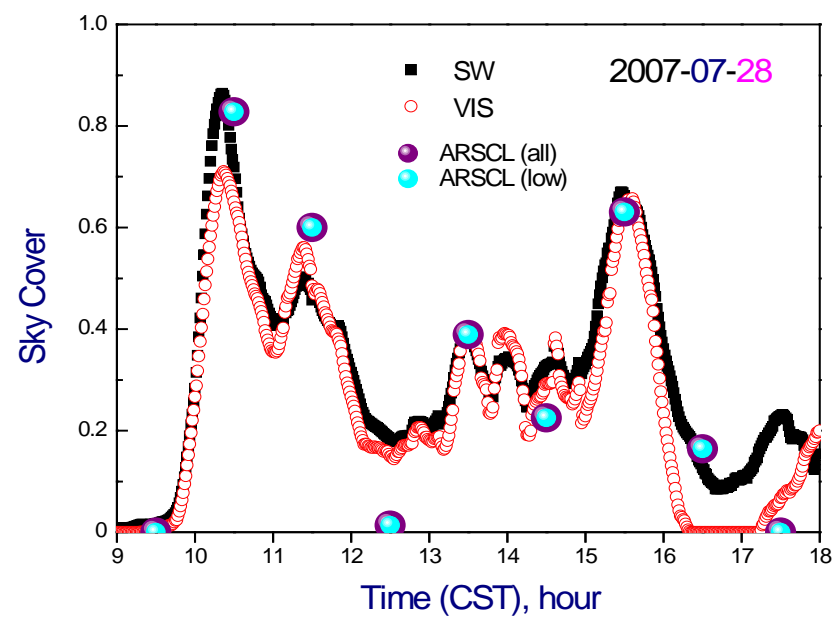

Fig. 5. Temporal realizations of the visible (red) and shortwave (black) fractional sky cover for 28 July 2007. Purple and cyan solid circles represent the hourly-averaged ARSCL nadir-view cloud fraction for all and low clouds, respectively.

$0.01)$. Thus, this value $(\sim 0.01)$ is less than those obtained for clear-sky conditions $(\sim 0.1)$ by a factor of 10 . This confirms that the cloudy-sky fluxes have weak spectral changes (Sect. 2). Thus, Eq. (3) can be used for estimating fractional sky cover. We emphasize that Eq. (3) should be applied for time periods where the difference of observed diffuse fluxes $F(500)-F(415)$ is negative. If this difference is positive, $N_{\text {vis }}$ is assumed to be 1 .

\section{Results}

To evaluate our technique, we apply independent data obtained by the well-established empirical method (Long et al., 2006) that uses all-sky shortwave fluxes measured by a ground-based pyranometer. Typically, observations made on a cloud-free day in close temporal proximity of a given cloudy day are applied for obtaining the corresponding "clear-sky" fluxes for estimation of the shortwave fractional sky cover $N_{\mathrm{SW}}$. These "clear-sky" fluxes are those that would be measured by the pyranometer if clouds were not present during observations. At the ACRF site, the pyranometer is located near the MFRSR and their separation is about $20 \mathrm{~m}$. Since $N_{\mathrm{SW}}$ values are obtained by a well-established method, they can be used to check the MFRSR-based $N_{\text {vis }}$ for consistency. To smooth out short-term fluctuations of the fractional sky cover, we apply a moving average approach (21point window). Also, we add time series of the ARSCLbased nadir-view cloud fraction. In particular, 1-h averaged ARSCL-based cloud fractions NARSCL for low (cloud base is less than $3 \mathrm{~km}$ ) and for all clouds are incorporated.. Recall that $N_{\mathrm{SW}}$ and $N_{\text {vis }}$ represent hemispherical observations, while $N_{\mathrm{ARSCL}}$ characterizes the zenith pointing measurements. We use the ARSCL-based properties (cloud fraction 

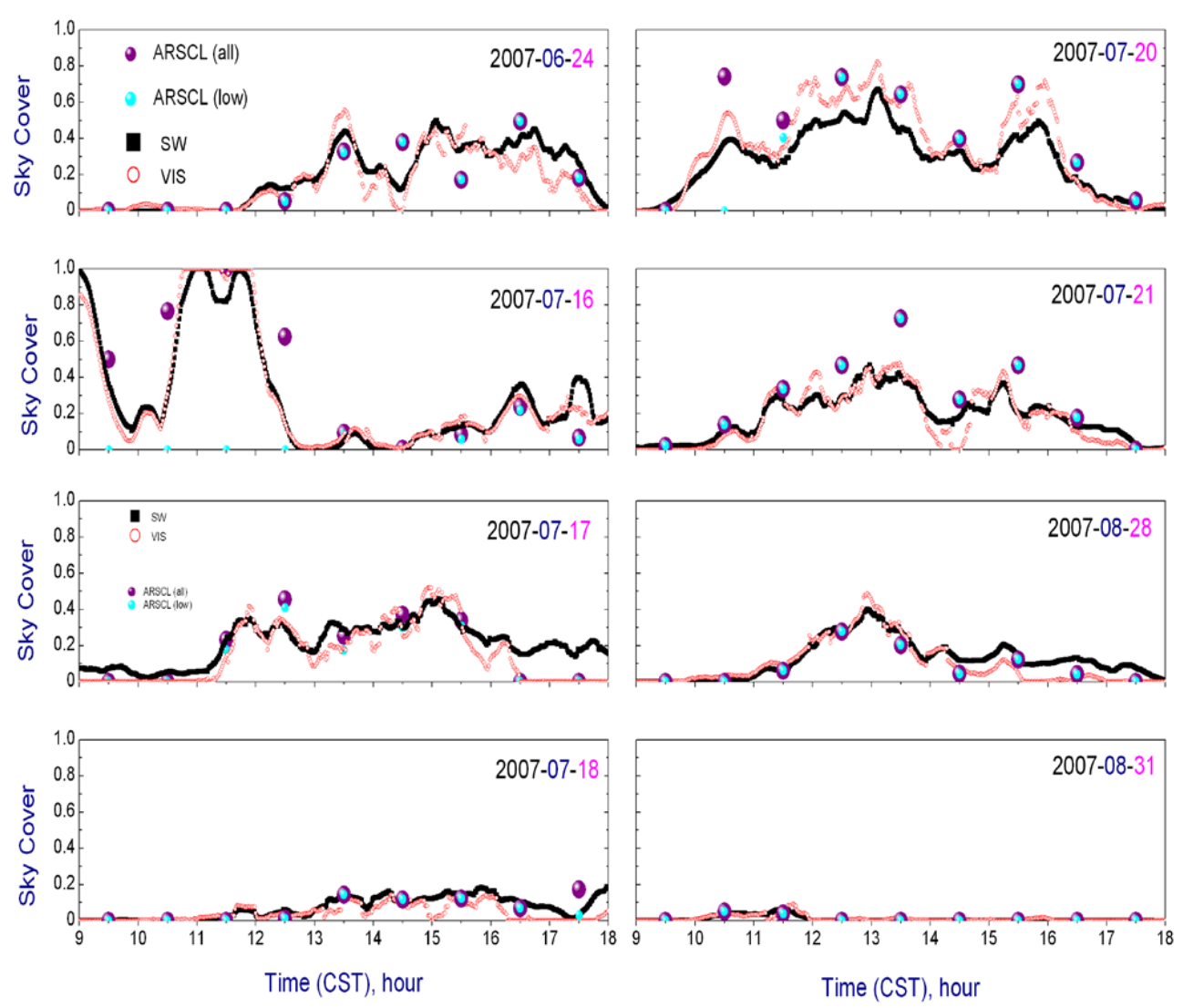

Fig. 6. Same as Fig. 5 but for 8 days.

versus cloud base) and the TSI images to illustrate how the vertical stratification of clouds and their horizontal distribution over a large area neighboring the ACRF site could contribute to the differences between $N_{\mathrm{SW}}$ and $N_{\text {vis }}$.

We start with comparison of $N_{\mathrm{SW}}$ and $N_{\text {vis }}$ for a day with low clouds only (Fig. 5). A reasonable agreement between $N_{\mathrm{SW}}$ and $N_{\text {vis }}$ is obtained for most of the day (from 09:00 to 16:00 CST). However, a relatively large difference between $N_{\mathrm{SW}}$ and $N_{\text {vis }}$ occurs in the evening (from 16:00 to 18:00 CST). Are these differences associated with a relative position of clouds in the sky (hemispherical FOV) and their type/abundance? Unfortunately, the TSI images are not available for this day. To address this question, we provide similar comparison for other 8 days with lidar data (Fig. 2) and TSI (Fig. 3) images. For example, a similar large difference between $N_{\mathrm{SW}}$ and $N_{\text {vis }}$ is observed in the evening (from 16 to 18 ) for 17 July (Fig. 6), where $N_{\mathrm{SW}} \sim 0.2$ and $N_{\text {vis }}$ is zero. The corresponding TSI image includes a few optically thin clouds near the edge (Fig. 3b). Thus, the MFRSR-based method underestimates slightly the fractional sky cover for this time period. Let us consider another example with clearsky conditions observed in 28 August at 17:00 CST, where $N_{\text {SW }} \sim 0.1$ and $N_{\text {vis }}$ is zero (Fig. 6). The corresponding TSI image does not include any clouds (Fig. 3b). Therefore, the pyranometer-based method overestimates slightly the fractional sky cover for this time period. However, both the MFRSR- and pyranometer-based methods are able to provide a reasonable estimation of the fractional sky cover for 31 August at 11:30 CST (Fig. 6) where a few optically thin clouds are observed (Fig. 3b).

For the majority of cases considered here, clouds are located below $3 \mathrm{~km}$ (Fig. 2). To illustrate the sensitivity of the differences between $N_{\mathrm{SW}}$ and $N_{\text {vis }}$ to the vertical stratification of clouds and their horizontal extent, we include in our analysis data obtained for 16 July when mid-latitude (cloud base $\sim 4 \mathrm{~km}$ ) optically thin clouds almost completely cover the sky in the morning and noon (from 09:00 to 12:30 CST) and low-latitude cumuli occur in the afternoon (Fig. 2). In general, the cumuli are small (Fig. 3a). Both the MFRSRand pyranometer-based methods capture the corresponding large diurnal changes of the fractional sky cover of mid- and low-latitude clouds, and time series of $N_{\mathrm{SW}}$ and $N_{\text {vis }}$ correlate reasonably well (Fig. 6). However, substantial differences between $N_{\mathrm{SW}}$ and $N_{\text {vis }}$ occur for some time instances (e.g., at 17:30 CST). Below, we outline potential reasons for these observed differences.

Both $N_{\text {vis }}$ and $N_{\mathrm{SW}}$ represent a hemispherical measure of cloud amount, and this measure is quite sensitive to a cloud 
location within hemispherical FOV. This sensitivity is more pronounced for clouds with small horizontal extent, such as cumuli (Kassianov et al., 2005a). For example, cloud chord length (CCL) is applied typically to characterize a representative horizontal scale of the broken clouds. We define the CCL as the length of time that an individual cloud is over a ground-based zenith-pointing instrument multiplied by the wind speed at cloud base, and found that clouds with smallest CCL (less than or equal to $0.1 \mathrm{~km}$ ) are the most frequent (Berg and Kassianov, 2008). Similar results are obtained for marine cumuli (e.g., Koren et al., 2008). A relatively small cloud, which partially covers the FOV, can be viewed very differently by two separated instruments (Kassianov et al., 2005a, b). For example, the same cloud could be located in a center of MFRSR-related FOV and near to edge of pyranometer-related FOV, and vice versa. Cases with "center"- and "edge"-type cloud location are characterized by large and small values of fractional sky cover, respectively (Kassianov et al., 2005a). For such instances, the MFRSRand pyranometer-based estimations of fractional sky cover are expected to be different.

In addition to the issues associated with instrument separation and the small-scale variability of cumuli, other factors can contribute to the observed differences between the visible $N_{\text {vis }}$ and shortwave $N_{\text {SW }}$ values (Figs. 5, 6). These factors include the differences in inputs for the two methods considered here (spectrally resolved fluxes versus broadband fluxes) and the diurnal variations of atmospheric aerosols observed during a cloudy day. These variations are neglected by the empirical method $\left(N_{\mathrm{SW}}\right)$ and are incorporated in the physically-based approach $N_{\text {vis }}$ described here. Despite effects associated with these factors, the temporal variations of $N_{\text {vis }}$ are $N_{\text {SW }}$ are in a good agreement (Fig. 5, 6). As a result, a strong linear relationship between $N_{\text {vis }}$ and $N_{\mathrm{SW}}$ is obtained (Fig. 7a). For the majority of cases, points cluster tightly around the slope (Fig. 7a), and the difference between $N_{\text {vis }}$ and $N_{\text {SW }}$ is less than 0.1 (Fig. 7b).

\section{Summary}

We describe a new method for estimating the fractional sky cover $N_{\text {vis }}$ by using the diffuse all-sky surface irradiances measured at two close wavelengths in the visible spectral range and their clear-sky counterparts provided by a physically-based approach (Kassianov et al., 2011). The aerosol optical properties (aerosol optical depth, singlescattering albedo and asymmetry parameter) obtained for cloud-free time periods and their temporal interpolation form the basis of this approach. To illustrate the performance of this method, we apply high-temporal resolution data from a ground-based Multi-Filter Rotating Shadowband Radiometer (MFRSR) collected during identified 13 days identified with cumuli observed in the summer of 2007 at the US Department of Energy Atmospheric Radiation Measurement

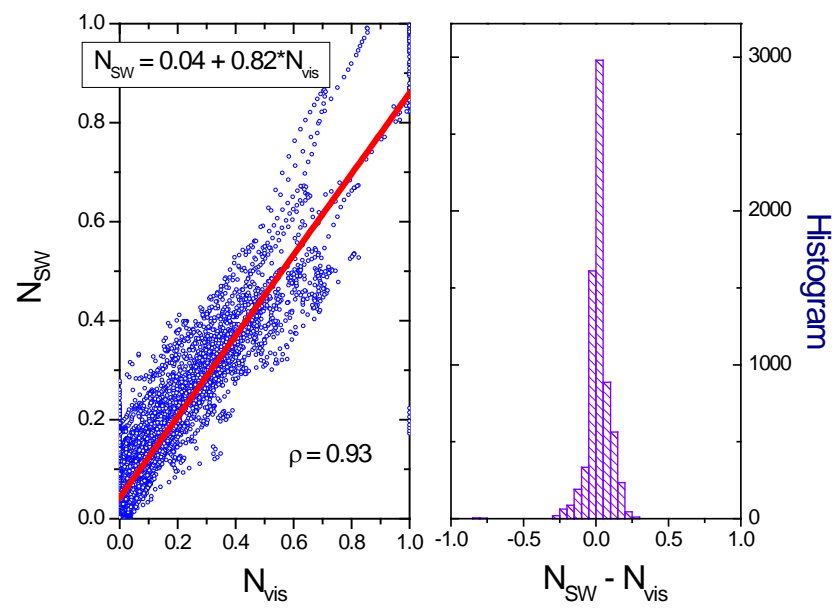

Fig. 7. The visible versus shortwave fractional sky cover (left) and the corresponding difference (right) for the selected 13 cloudy days.

(ARM) Climate Research Facility Southern Great Plains (SGP) site.

The MFRSR provides the total all-sky surface downwelling irradiance and its diffuse and direct components at six wavelengths of $415,500,615,673,870$, and $940 \mathrm{~nm}$. For $N_{\text {vis }}$ estimation, we consider MFRSR data at two wavelengths (415 and $500 \mathrm{~nm}$ ) only. The MFRSR observations are accompanied by shortwave measurements from a nearby broadband pyranometer. These shortwave measurements together with a well-established method (Long et al., 2006) give us an independent estimation of fractional sky cover $N_{\text {SW }}$. We compare $N_{\text {vis }}$ with $N_{\text {SW }}$ and find a strong linear relationship between $N_{\text {vis }}$ and $N_{\mathrm{SW}}$ with a large correlation coefficient (0.9). Also, we demonstrate that the difference between $N_{\text {vis }}$ and $N_{\mathrm{SW}}$ is less than 0.1 for the majority of cases $(\sim 80 \%)$. The favorable agreement ( $N_{\text {vis }}$ vs. $\left.N_{\text {SW }}\right)$ suggests that our method based on the spectrally resolved irradiances can be applied for estimation of the fractional sky cover for different cloud types, including cumuli. We plan to repeat this study for additional cases representing the natural variability of atmospheric conditions (clouds, aerosol) over a wide range of geographic locations.

The MFRSR data have been used successfully to examine changes of water vapor (Alexandrov et al., 2009), aerosol optical, and microphysical properties (Harrison and Michalsky, 1994; Alexandrov et al., 2002; Kassianov et al., 2007; Michalsky et al., 2010), cloud optical depth and droplet effective radius (Min and Harrison, 1996), and the fractional sky cover of optically thick clouds with large horizontal size (Min et al., 2008). The method described here extends the capabilities of the MFRSR observations by offering an opportunity to sample the fractional sky cover of optically thin clouds with small horizontal size, such as shallow cumuli. Thus, the worldwide deployed MFRSRs can supply an integrated dataset of the water vapor, aerosol and cloud 
properties, and unique MFRSR-based datasets could be developed for different locations. Such datasets together with others from ground- and satellite-based observations can be applied to improve the understanding of the complex aerosolcloud interactions, including the relationship between the fractional sky cover and aerosol loading and absorption.

Acknowledgements. This work has been supported by the Office of Biological and Environmental Research (OBER) of the US Department of Energy (DOE) as part of the Atmospheric Radiation Measurement (ARM), and Atmospheric Systems Research (ASR) Programs. The Pacific Northwest National Laboratory (PNNL) is operated by Battelle for the DOE under contract DE-AC06-76RLO 1830. The authors are grateful to three reviewers for the valuable comments and constructive suggestions.

Edited by: A. Macke

\section{References}

Alexandrov, M. D., Lacis, A., Carlson, B. E., and Cairns, B.: Remote sensing of atmospheric aerosols and trace gases by means of Multifilter Rotating Shadowband Radiometer, Part II: climatological applications, J. Atmos. Sci., 59, 544-566, 2002.

Alexandrov, M. D., Schmid, B., Turner, D. D., Cairns, B., Oinas, V., Lacis, A. A., Gutman, S. I., Westwater, E. R., Smirnov, A., and Eilers, J.: Columnar water vapor retrievals from multifilter rotating shadowband radiometer data, J. Geophys. Res., 114, D02306, doi:10.1029/2008JD010543, 2009.

Berg, L. K and Kassianov, E. I.: Temporal variability of fair-weather cumulus statistics at the ACRF SGP site, J. Climate, 21, 33443358, 2008.

Berg, L. K., Kassianov, E., Long, C. N., and Mills Jr., D. L.: Surface summertime radiative forcing by shallow cumuli at the ARM SGP, J. Geophys. Res., 116, D01202, doi:10.1029/2010JD014593, 2011.

Dong, X., Xi, B., and Minnis, P.: A climatology of midlatitude continental clouds from the ARM SGP Central Facility. Part II: Cloud fraction and surface radiative forcing, J. Climate, 19, 1765-1783, 2006.

Dupont, J.-C., Haeffelin, M., and Long, C. N.: Evaluation of cloudless-sky periods detected by shortwave and longwave algorithms using lidar measurements, Geophys. Res. Lett., 35, L10815, doi:10.1029/2008GL033658, 2008.

Hahn, C., Rossow, W., and Warren, S.: ISCCP cloud properties associated with standard cloud types identified in individual surface observation. J. Climate, 14, 11-28, 2001.

Harrison, L. and Michalsky, J.: Objective algorithms for the retrieval of optical depths from ground-based measurements, J. Appl. Opt., 22, 5126-5132, 1994.

Kassianov, E. and Kogan, Y. L.: Spectral dependence of radiative horizontal transport in stratocumulus clouds and its effect on near-IR absorption, J. Geophys. Res., 107(D23), 4712, doi:10.1029/2002JD002103, 2002.

Kassianov, E., Long, C. N., and Ovtchinnikov, M.: Cloud sky cover versus cloud fraction: whole-sky simulations and observations, J. Appl. Meteorol., 44, 86- 98, 2005a.

Kassianov, E., Long, C. N., and Christy, J.: Cloud-base-height estimation from paired ground-based hemispherical observations, J. Appl. Meteor., 44, 1221-1233, 2005 b.
Kassianov, E., Flynn, C. J., Ackerman, T. P., and Barnard, J. C.: Aerosol single-scattering albedo and asymmetry parameter from MFRSR observations during the ARM Aerosol IOP 2003, Atmos. Chem. Phys., 7, 3341-3351, doi:10.5194/acp-7-3341-2007, 2007.

Kassianov, E., Barnard, J., Berg, L. K., Long, C. N., and Flynn, C.: Shortwave Spectral Radiative Forcing of Cumulus Clouds from Surface Observations, Geophys. Res. Lett.,38, L07801, doi:10.1029/2010GL046282, 2011.

Kaufman, Y. J. and Koren, I.: Smoke and pollution aerosol effect on cloud cover, Science, 313, 655-658, 2006.

Koren, I., Oreopoulos, L., Feingold, G., Remer, L. A., and Altaratz, O.: How small is a small cloud?, Atmos. Chem. Phys., 8, 38553864, doi:10.5194/acp-8-3855-2008, 2008.

Lane, D. E., Goris, K., and Somerville, R. C. J.: Radiative transfer through broken clouds: Observations and model validation, J. Climate, 15, 2921-2933, 2002.

Long, C. N., Ackerman, T. P., Gaustad, K. L., and Cole, J. N. S.: Estimation of fractional sky cover from broadband shortwave radiometer measurements, J. Geophys. Res., 111, D11204, doi:10.1029/2005JD006475, 2006.

McComiskey, A., Schwartz, S. E., Schmid, B., Guan, H., Lewis, E. R., Ricchiazzi, P., and Ogren, J. A.: Direct aerosol forcing: Calculation from observables and sensitivities to inputs, J. Geophys. Res., 113, D09202, doi:10.1029/2007JD009170, 2008.

Michalsky, J., Denn, F., Flynn, C., Hodges, G., Kiedron, P., Koontz, A., Schlemmer, J., and Schwartz, S. E.: Climatology of aerosol optical depth in north-central Oklahoma: 1992-2008, J. Geophys. Res., 115, D07203, doi:10.1029/2009JD012197, 2010.

Min, Q. and Harrison, L. C.: Cloud properties derived from surface MFRSR measurements and comparison with GOES results at the ARM SGP Site, Geophys. Res. Lett., 23, 1641-1644, doi:10.1029/96GL01488, 1996.

Min, Q., Wang, T., Long, C. N., and Duan, M.: Estimating fractional sky cover from spectral measurements,' J. Geophys. Res., 113, D20208, doi:10.1029/2008JD010278, 2008.

Perlwitz, J. and Miller, R. L.: Cloud cover increase with increasing aerosol absorptivity: A counterexample to the conventional semidirect aerosol effect, J. Geophys. Res., 115, D08203, doi:10.1029/2009JD012637, 2010.

Quaas, J., Stevens, B., Stier, P., and Lohmann, U.: Interpreting the cloud cover - aerosol optical depth relationship found in satellite data using a general circulation model, Atmos. Chem. Phys., 10, 6129-6135, doi:10.5194/acp-10-6129-2010, 2010.

Slingo, A., Ackerman, T. P., Allan, R. P., Kassianov, E., McFarlane, S. A., Robinson, G. J., Barnard, J. C., Miller, M. A., Harries, J. E., Russell, J. E., and Dewitte, S.: Observations of the impact of a major Saharan dust storm on the atmospheric radiation balance, Geophys. Res. Lett., 33, L24817, doi:10.1029/2006GL027869, 2006.

Su, W., Loeb, N. G., Xu, K. M., Schuster, G. L., and Eitzen, Z. A.: An estimate of aerosol indirect effect from satellite measurements with concurrent meteorological analysis, J. Geophys. Res., 115, D18219, doi:10.1029/2010JD013948, 2010.

Zhang, Y., Long, C. N., Rossow, W. B., and Dutton, E. G.: Exploiting diurnal variations to evaluate the ISCCP-FD flux calculations and radiative-flux-analysis-processed surface observations from BSRN, ARM, and SURFRAD, J. Geophys. Res., 115, D15105, doi:10.1029/2009JD012743, 2010. 